\title{
Statistical-mechanical analysis of adaptive filter with clipping saturation-type nonlinearity
}

This paper was downloaded from TechRxiv (https://www.techrxiv.org).

\section{LICENSE}

CC BY 4.0

SUBMISSION DATE / POSTED DATE

$13-10-2021 / 03-02-2022$

\section{CITATION}

Miyoshi, Seiji (2021): Statistical-mechanical analysis of adaptive filter with clipping saturation-type nonlinearity. TechRxiv. Preprint. https://doi.org/10.36227/techrxiv.16801336.v2

$\mathrm{DOI}$

10.36227/techrxiv.16801336.v2 


\title{
Statistical-mechanical analysis of adaptive filter with clipping saturation-type nonlinearity
}

\author{
Seiji Miyoshi, Senior Member, IEEE
}

\begin{abstract}
In most practical adaptive signal processing systems, e.g., active noise control, active vibration control, and acoustic echo cancellation, substantial nonlinearities that cannot be neglected exist. In this paper, we analyze the behaviors of an adaptive system in which the output of the adaptive filter has the clipping saturation-type nonlinearity by a statistical-mechanical method. We discuss the dynamical and steady-state behaviors of the adaptive system by asymptotic analysis, steady-state analysis, and numerical calculation. As a result, it has become clear that the saturation value has the critical point at which the system's mean-square stability and instability switch. The obtained theory well explains the strange behaviors around the critical point observed in the computer simulation. Finally, the exact value of the critical point is also derived.
\end{abstract}

Index Terms-adaptive filter, adaptive signal processing, system identification, LMS algorithm, clipping saturation-type nonlinearity, piecewise linearity, statistical-mechanical analysis

\section{INTRODUCTION}

A DAPTIVE signal processing is used in wide areas such as communication systems and acoustic systems [1], [2]. Active noise control (ANC) [3]-[6], active vibration control (AVC) [7], acoustic echo cancellation [8], and system identification [9] are examples of specific applications of adaptive signal processing. Adaptive signal processing using linear filters has been theoretically analyzed [1], [2].

In most practical adaptive systems, for example, power amplifiers and transducers such as loudspeakers and microphones, substantial nonlinearities that cannot be neglected exist [1], [2]. Such nonlinearities are inevitable and it is extremely important to investigate in detail their effects on the overall performance of adaptive systems. Therefore, there have been many studies on adaptive signal processing systems including nonlinear components [10]-[31]. In some of these studies, nonlinearities where an input signal and an error signal are expressed by their signs $( \pm 1)$ or three values $(-1,0,+1)$ have been investigated [10]-[20]. Note, however, that such nonlinearities are rather intended to reduce computational complexity. Bershad [21] analyzed the case in which the update by the least-mean-square (LMS) algorithm [32] has $\left(1-e^{-a x}\right)$ saturation-type nonlinearity, assuming a small step size. Costa et al. [22] analyzed the case in which the output of the adaptive filter has an error function (erf) saturationtype nonlinearity, assuming a small step size. Costa et al. [23],

Manuscript received April 19, 202x; revised December 11, 202x. This work was supported by JSPS KAKENHI Grant Number JP20K04494.

S. Miyoshi is with the Department of Electrical, Electronic and Information Engineering, Faculty of Engineering Science, Kansai University, 3-335 Yamate-cho, Suita-shi, Osaka 564-8680, Japan (e-mail: miyoshi@kansaiu.ac.jp).
[24] analyzed the ANC in which the secondary path has an erf saturation-type nonlinearity. Snyder and Tanaka [25] proposed the replacement of the finite-duration impulse response (FIR) filter with a neural network to deal with the primary path nonlinearity in ANC/AVC. Costa [26] analyzed a hearing aid feedback canceller with an erf saturation-type nonlinearity. Costa et al. [27] analyzed the model in which the output of the adaptive filter has a dead-zone nonlinearity caused by a class B amplifier or a nonlinear actuator, assuming a small step size. Tobias and Seara [28] analyzed the behaviors of the modified LMS algorithm derived from the improved cost function in the case where the output of the adaptive filter has an erf saturation-type nonlinearity. Bershad [29] analyzed the case where the update by the LMS algorithm has an erf saturation-type nonlinearity and extended the analysis to the case of tracking a Markov channel in the context of system identification. As described so far, there have been many studies on an erf saturation-type nonlinearity of adaptive systems. On the other hand, Hamidi et al. [30] reported their analysis, computer simulation, and experimental results of the ANC model in which the output of the adaptive filter has the clipping saturation-type nonlinearity. They proposed the modification of the cost function to avoid using a nonlinear region to improve the adaptive algorithm. Stenger and Kellermann [31] proposed the use of clipping-type preprocessing in adaptive echo cancellation to cancel the effect of nonlinear echo paths.

On the other hand, our group has recently studied the application of the statistical-mechanical method [33] to the analysis of adaptive signal processing. In the statistical-mechanical method, the universal properties of a system consisting of many microscopic variables are discussed macroscopically and deterministically by introducing a small number of macroscopic variables and considering the large-system limit. Therefore, the method is particularly well suited for the analysis of signal processing that involves an adaptive filter with a large tap length. Our group [34], [35] has also analyzed feed-forward ANC updated by the Filtered-x LMS (FXLMS) algorithm using the statistical-mechanical method. However, the analyses dealt with the case where the primary path, secondary path, and adaptive filter were all linear. Our group [36] has also analyzed the model in which both the unknown system and adaptive filter have the Volterra-type nonlinearity [37] as an application of the statistical-mechanical method to nonlinear adaptive signal processing. Although Volterra filters have nonlinear characteristics, it was relatively easy to apply the statistical-mechanical method used for linear systems to their analysis. However, it was only applicable to Volterra filters of a specific order. Moreover, in that previous study, 
we did not deal with simple nonlinearities such as saturation and dead-zone types, which are often found in actual adaptive processing systems.

As described above, although there have been many studies on adaptive signal processing with nonlinear components, there are only a few studies on clipping saturation-type nonlinearity; in particular, there have been no analytical studies as far as we searched the literature. The clipping saturation type, i.e., the piecewise linear type, seems to be the most direct expression of the saturation phenomenon of components constituting adaptive systems such as power amplifiers and transducers such as loudspeakers and microphones. The erftype saturation function, which has been well analyzed in previous studies, is rather an approximation of the clipping saturation-type saturation function. In the paper [27] on the analysis of the dead-zone type rather than the saturation type, Costa et al. stated the following: "To facilitate the development of analytical models, it is convenient to approximate the piecewise nonlinearity by a continuous and more mathematically tractable function" and "The error function has been successfully used as an approximation of a saturationtype nonlinearity." Moreover, Costa et al. [27] compared the results of the erf-type analysis with the results of computer simulations performed with the piecewise linearity, indicating that it is indeed desirable to analyze nonlinearity expressed as the piecewise linearity rather than nonlinearity approximated by the erf type. In light of these developments, in this paper, we analyze the behaviors of a system with an adaptive filter whose output has clipping saturation-type nonlinearity, i.e., piecewise linearity.

The rest of this paper is organized as follows. In Sec. II, we define the model analyzed in this study. In Sec. III, we describe the statistical-mechanical analysis for the model in detail. In Sec. IV, we demonstrate the validity of the obtained theory by comparing theoretical results with simulation results. In that section, we also clarify that there exists the critical value $S_{C}$ for the saturation value $S$ at which the properties of the adaptive system switch markedly. In addition, some results obtained by the steady-state, asymptotic, and numerical analyses are shown. Furthermore, we obtain the exact value of $S_{C}$ by asymptotic analysis. In Sec. V we conclude our study in this paper.

\section{MODEL}

Figure 1 shows a block diagram of the adaptive system analyzed in this paper. The impulse response of the unknown system $\mathrm{G}$ is an $M$-dimensional arbitrary vector

$$
\boldsymbol{g}_{0}=\left[g_{1}, g_{2}, \ldots, g_{M}\right]^{\top},
$$

and is time-invariant. Here, ${ }^{\top}$ denotes the transpose of a vector. The adaptive filter $\mathrm{W}$ is an $N$-tap FIR filter. Its coefficient vector is

$$
\boldsymbol{w}(n)=\left[w_{1}(n), w_{2}(n), \ldots, w_{N}(n)\right]^{\top},
$$

where $n$ denotes the time step. All initial values $w_{i}(0), i=$ $1, \ldots, N$, of the coefficients are assumed to be zero. We

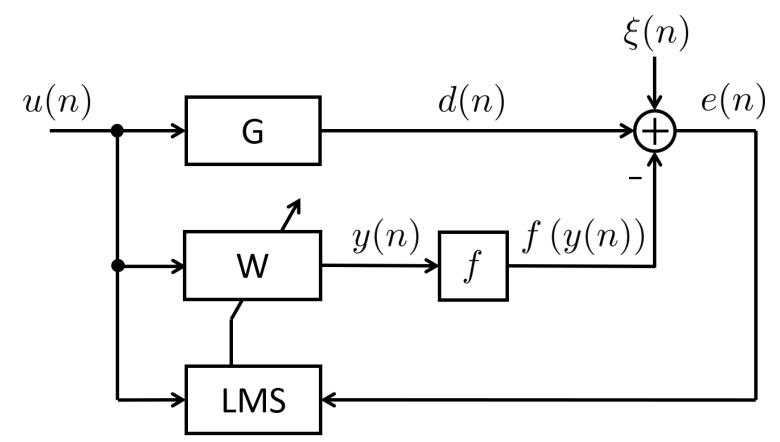

Fig. 1: Block diagram of the adaptive system.

assume that the tap length $N$ of the adaptive filter $\mathrm{W}$ is set to satisfy

$$
N \geq M .
$$

Also, let $\boldsymbol{g}$ be a vector made into $N$ dimensions by adding $N-M$ zeros to $\boldsymbol{g}_{0}$. That is,

$$
\begin{aligned}
\boldsymbol{g} & =\left[g_{1}, g_{2}, \ldots, g_{M}, g_{M+1}, \ldots, g_{N}\right]^{\top}, \\
g_{i} & =0, \quad i=M+1, \ldots, N .
\end{aligned}
$$

Note that while it is assumed in most previous studies assume that the dimensions of $\boldsymbol{g}_{0}$ and $\boldsymbol{w}$ are the same [22]-[24], [27][29], our model essentially allows for arbitrary $\boldsymbol{g}_{0}$ and does not make strict assumptions on its dimension $M$ or its elements $\left\{g_{i}\right\}, i=1, \ldots, M$.

We define the parameter $\sigma_{g}^{2}$ as

$$
\sigma_{g}^{2} \triangleq \frac{1}{N}\left\|\boldsymbol{g}_{0}\right\|_{2}^{2}=\frac{1}{N}\|\boldsymbol{g}\|_{2}^{2}=\frac{1}{N} \sum_{i=1}^{N} g_{i}^{2} .
$$

As will become clear later, our theory depends on the unknown system $\mathrm{G}$ only via $\sigma_{g}^{2}$. To demonstrate this, we will show in IV. RESULTS AND DISCUSSION that the theory is valid for actual $\boldsymbol{g}_{0}$ measured experimentally.

The input signal $u(n)$ is assumed to be independently drawn from a distribution with

$$
\langle u(n)\rangle=0, \quad\left\langle u(n)^{2}\right\rangle=\sigma^{2},
$$

where $\langle\cdot\rangle$ denotes expectation. That is, the input signal is white. Although the assumption that the input signal is white may seem to be a major constraint in this analysis, the white input model is an important part of practical applications, especially in system identification, and provides a clear insight into the behavior of the algorithm. Moreover, the white input case provides a baseline for other cases [22]. The tap input vector $\boldsymbol{u}(n)$ at time step $n$ is

$$
\boldsymbol{u}(n)=[u(n), u(n-1), \ldots, u(n-N+1)]^{\top} .
$$

Note that only the mean and variance of the distribution are specified in (7). No specific distributions, for example, the Gaussian distribution, are assumed.

Outputs of the unknown system $\mathrm{G}$ and the adaptive filter $\mathrm{W}$ are convolutions of their own coefficients and a sequence of 
input signals. That is, the outputs $d(n)$ of $\mathrm{G}$ and $y(n)$ of $\mathrm{W}$ are respectively

$$
\begin{aligned}
& d(n)=\boldsymbol{g}^{\top} \boldsymbol{u}(n)=\sum_{i=1}^{N} g_{i} u(n-i+1), \\
& y(n)=\boldsymbol{w}(n)^{\top} \boldsymbol{u}(n)=\sum_{i=1}^{N} w_{i}(n) u(n-i+1) .
\end{aligned}
$$

The nonlinearity of the adaptive filter $\mathrm{W}$ is modeled by the function $f$ placed after $\mathrm{W}$. The function $f$ represents the clipping saturation-type nonlinearity and is expressed as

$$
f(x)= \begin{cases}S, & x>S \\ -S, & x<-S, \\ x, & \text { otherwise }\end{cases}
$$

where $S$ is a nonnegative real number. Figure 2 shows the function $f$.

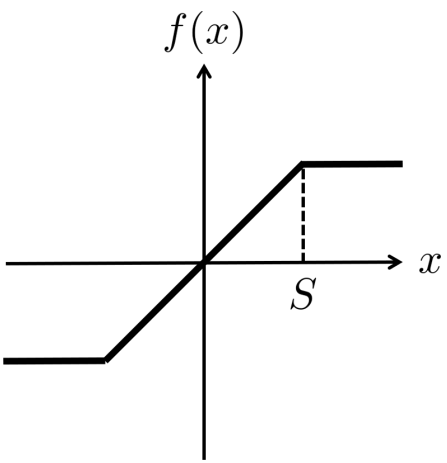

Fig. 2: Clipping saturation-type nonlinearity.

The error signal $e(n)$ is generated by adding an independent background noise $\xi(n)$ to the difference between $d(n)$ and $y(n)$. That is,

$$
e(n)=d(n)-f(y(n))+\xi(n) .
$$

Here, the mean and variance of $\xi(n)$ are zero and $\sigma_{\xi}^{2}$, respectively.

The LMS algorithm [32] is used to update the adaptive filter. That is,

$$
\boldsymbol{w}(n+1)=\boldsymbol{w}(n)+\mu e(n) \boldsymbol{u}(n),
$$

where $\mu$ is a positive real number and is called the step size.

\section{ANALYSIS}

In this section, we theoretically analyze the behaviors of the adaptive system with clipping saturation-type nonlinearity by the statistical-mechanical method. From (12), the MSE is expressed as

$$
\begin{aligned}
\left\langle e^{2}\right\rangle & =\left\langle(d-f(y)+\xi)^{2}\right\rangle \\
& =\left\langle d^{2}\right\rangle+\left\langle f(y)^{2}\right\rangle-2\langle d f(y)\rangle+\sigma_{\xi}^{2} .
\end{aligned}
$$

In this section, we omit the time step $n$ unless otherwise stated to avoid a rather cumbersome notation. We assume $N \rightarrow \infty^{1}$

\footnotetext{
${ }^{1}$ This is called the thermodynamic limit in statistical mechanics.
}

while keeping both $\sigma_{g}^{2}$ and

$$
\rho^{2} \triangleq N \sigma^{2}
$$

constant, in accordance with the statistical-mechanical method [33]. Note that when we set $\rho^{2}=1$, it corresponds to the normalized LMS (NLMS) algorithm. Then, from the central limit theorem, both $d$ and $y$ are stochastic variables that obey the Gaussian distribution. Their means are zeros, and the variance-covariance matrix is

$$
\boldsymbol{\Sigma}=\rho^{2}\left(\begin{array}{cc}
\sigma_{g}^{2} & r \\
r & Q
\end{array}\right) .
$$

Here, $Q$ and $r$ are macroscopic variables that are respectively defined as

$$
\begin{gathered}
Q \triangleq \frac{1}{N} \boldsymbol{w}^{\top} \boldsymbol{w}, \\
r \triangleq \frac{1}{N} \boldsymbol{g}^{\top} \boldsymbol{w} .
\end{gathered}
$$

The derivation of the means and variance-covariance matrix is given in detail in Appendix A.

We obtain three sample means in (15) as follows by carrying out the Gaussian integration for $d$ and $y$ :

$$
\begin{aligned}
\left\langle d^{2}\right\rangle= & \rho^{2} \sigma_{g}^{2}, \\
\left\langle f(y)^{2}\right\rangle= & S^{2}+\left(\rho^{2} Q-S^{2}\right) \operatorname{erf}\left(\frac{S}{\sqrt{2 \rho^{2} Q}}\right) \\
& -S \sqrt{\frac{2 \rho^{2} Q}{\pi}} \exp \left(-\frac{S^{2}}{2 \rho^{2} Q}\right), \\
\langle d f(y)\rangle= & \rho^{2} r \operatorname{erf}\left(\frac{S}{\sqrt{2 \rho^{2} Q}}\right),
\end{aligned}
$$

where $\operatorname{erf}(\cdot)$ is an error function defined as

$$
\operatorname{erf}(x) \triangleq \frac{2}{\sqrt{\pi}} \int_{0}^{x} \exp \left(-t^{2}\right) \mathrm{d} t .
$$

Equation (20) is easily derived from (17). Equations (21) and (22) are derived in detail in Appendices B and C, respectively.

From (15), (20), (21), and (22), we obtain the MSE as

$$
\begin{aligned}
\left\langle e^{2}\right\rangle= & \rho^{2} \sigma_{g}^{2}+S^{2}+\left(\rho^{2} Q-2 \rho^{2} r-S^{2}\right) \operatorname{erf}\left(\frac{S}{\sqrt{2 \rho^{2} Q}}\right) \\
& -S \sqrt{\frac{2 \rho^{2} Q}{\pi}} \exp \left(-\frac{S^{2}}{2 \rho^{2} Q}\right)+\sigma_{\xi}^{2} .
\end{aligned}
$$

This formula shows that the MSE is a function of the macroscopic variables $Q$ and $r$. Therefore, we derive differential equations that describe the dynamical behaviors of these variables in the following. Multiplying both sides of (13) on the left by $\boldsymbol{g}^{\top}$ and using (19), we obtain

$$
N r(n+1)=N r(n)+\mu e(n) d(n) .
$$

We introduce time $t$ defined by

$$
t \triangleq n / N,
$$

and use it to represent the adaptive process. Then, $t$ becomes a continuous variable since the limit $N \rightarrow \infty$ is considered. 
These calculations are in line with the statistical-mechanical analysis of online learning [38].

If the adaptive filter is updated $N \mathrm{~d} t$ times in an infinitely small time $\mathrm{d} t$, we can obtain $N \mathrm{~d} t$ equations as

$$
\begin{aligned}
& N r(n+1)=N r(n)+\mu e(n) d(n), \\
& N r(n+2)=N r(n+1)+\mu e(n+1) d(n+1) \text {, } \\
& N r(n+N \mathrm{~d} t)=N r(n+N \mathrm{~d} t-1) \\
& +\mu e(n+N \mathrm{~d} t-1) d(n+N \mathrm{~d} t-1) .
\end{aligned}
$$

Summing all these equations, we obtain

$$
N(r+\mathrm{d} r)=N r+N \mathrm{~d} t \mu\langle e d\rangle .
$$

Here, from the law of large numbers, we have represented the effect of the probabilistic variables by their means since the updates are executed $N \mathrm{~d} t$ times, that is, many times, to change $r$ by $\mathrm{d} r$. This property is called self-averaging in statistical mechanics [33]. From (12) and (30), we obtain a differential equation that describes the dynamical behavior of $r$ in a deterministic form as follows:

$$
\frac{\mathrm{d} r}{\mathrm{~d} t}=\mu\left(\left\langle d^{2}\right\rangle-\langle d f(y)\rangle\right) .
$$

Next, squaring both sides of (13) and proceeding in the same manner as for the derivation of the above differential equation for $r$, we can derive a differential equation for $Q$, which is given by

$$
\begin{aligned}
\frac{\mathrm{d} Q}{\mathrm{~d} t}= & \mu^{2}\left(\left\langle d^{2}\right\rangle-2\langle d f(y)\rangle+\left\langle f(y)^{2}\right\rangle+\sigma_{\xi}^{2}\right) \\
& +2 \mu(\langle d y\rangle-\langle y f(y)\rangle) .
\end{aligned}
$$

Equations (31) and (32) include five sample means. However, because three of the five means are already given in (20)-(22), we obtain the two remaining means as follows by carrying out the Gaussian integration for $d$ and $y$ :

$$
\begin{aligned}
\langle d y\rangle & =\rho^{2} r, \\
\langle y f(y)\rangle & =\rho^{2} Q \text { erf }\left(\frac{S}{\sqrt{2 \rho^{2} Q}}\right) .
\end{aligned}
$$

Equation (33) is easily derived from (17). Equation (34) is derived in detail in Appendix D.

Substituting (20)-(22), (33), and (34) into (31) and (32), we obtain the concrete formula of the simultaneous differential equations as follows:

$$
\begin{aligned}
\frac{\mathrm{d} r}{\mathrm{~d} t}= & \mu \rho^{2}\left(\sigma_{g}^{2}-r \operatorname{erf}\left(\frac{S}{\sqrt{2 \rho^{2} Q}}\right)\right) \\
\frac{\mathrm{d} Q}{\mathrm{~d} t}= & \mu \rho^{2}\left(\mu\left(\rho^{2} Q-2 \rho^{2} r-S^{2}\right)-2 Q\right) \operatorname{erf}\left(\frac{S}{\sqrt{2 \rho^{2} Q}}\right) \\
& -\mu^{2} \rho^{2} S \sqrt{\frac{2 \rho^{2} Q}{\pi}} \exp \left(-\frac{S^{2}}{2 \rho^{2} Q}\right) \\
& +\mu \rho^{2}\left(\mu\left(\rho^{2} \sigma_{g}^{2}+S^{2}+\sigma_{\xi}^{2}\right)+2 r\right) .
\end{aligned}
$$

We numerically solve the derived simultaneous differential equations, since they cannot be analytically solved. Substituting the obtained numerical solution into (24), we obtain the MSE learning curves.

From (6), (18), and (19), we can also obtain the meansquare deviation (MSD), or misalignment, as a function of the macroscopic variables $Q$ and $r$ as follows:

$$
\begin{aligned}
\mathrm{MSD} & =\|\boldsymbol{g}-\boldsymbol{w}\|_{2}^{2} \\
& =\|\boldsymbol{g}\|_{2}^{2}-2 \boldsymbol{g}^{\top} \boldsymbol{w}+\|\boldsymbol{w}\|_{2}^{2} \\
& =N\left(\sigma_{g}^{2}-2 r+Q\right) .
\end{aligned}
$$

Equation (39) shows that the MSD is proportional to the tap length $N$ in the model setting in this paper; therefore, we normalize the MSD by $N$ and call it the normalized MSD.

\section{RESULTS AND DISCUSSION}

\section{A. Learning Curves}

We first investigate the validity of the theory by comparing theoretical results with simulation results with regard to the dynamical behaviors of the MSE and normalized MSD, that is, the learning curves. Figures 3 and 4 show the learning curves obtained using the theory derived in the previous section, along with the corresponding simulation results. In these figures, "mu" denotes the step size $\mu$. In these figures, the curves represent theoretical results and the polygonal lines represent simulation results. In both the theoretical calculation and simulation, $\rho^{2}=\sigma_{g}^{2}=1$. In the theoretical calculation, the results are obtained by substituting $Q$ and $r$, which are respectively obtained by solving (35) and (36), into (24). Here, (35) and (36) are numerically solved by the RungeKutta method. In the computer simulations, the number of taps of the adaptive filter $\mathrm{W}$ is $N=400$ and ensemble means for 1000 trials are plotted. The impulse response $\boldsymbol{g}_{0}$ of the unknown system $G$ in all computer simulations in this paper was obtained experimentally by measurement [39] and is shown in Fig. 5. Its dimension $M$ is 256 . Note that $\boldsymbol{g}_{0}$ has been normalized to satisfy (6). All initial values $w_{i}(0), i=1, \ldots, N$ of the coefficients are set to zero in the simulation, and the initial condition $Q(0)=r(0)=0$ is used in the theoretical calculation.

Figures 3 and 4 show that the theory derived in this paper predicts the simulation results well in terms of average values. From Figs. 3a and 3b, it seems that the MSE almost converges at $t=50$ regardless of the step size $\mu$ for both $S=1$ and 3 . However, Fig. 4a shows that the normalized MSD continues to increase for $S=1$.

Next, we show the MSE at $t=10,100$, and 1000 in Fig. 6 to investigate the relationship between the saturation value $S$ and the MSE. In the computer simulations, the medians and standard deviations in 100 trials are plotted using error bars. Figures 6a-6c show that the MSE increases when $S$ is in the range of 1.1-1.3, and this tendency becomes obvious with time.

B. Critical value $S_{C}$ and steady-state analysis when $S>S_{C}$

To clarify the phenomenon of increasing MSE described in the previous subsection, we investigate the steady-state 


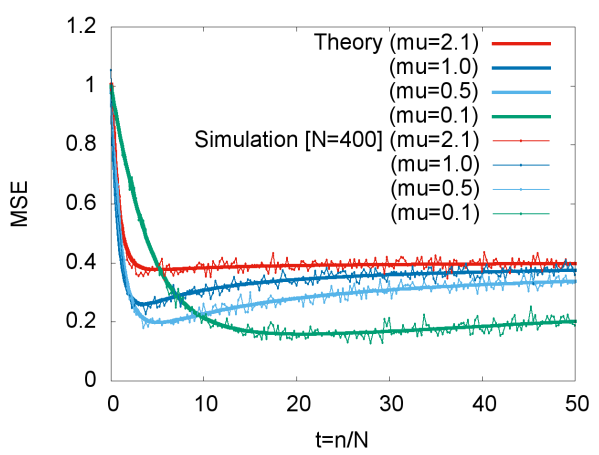

(a) $S=1$

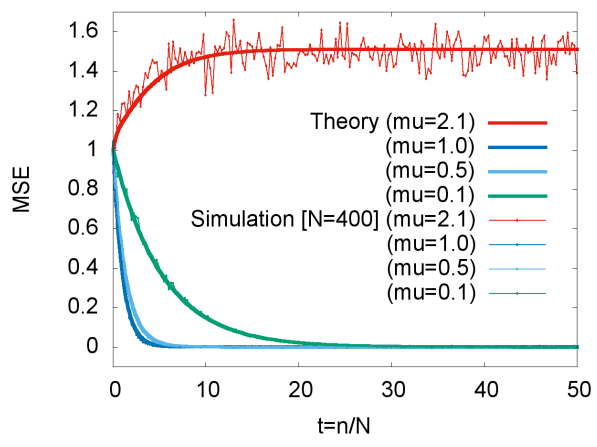

(b) $S=3$

Fig. 3: MSE learning curves $\left(\rho^{2}=\sigma_{g}^{2}=1, \sigma_{\xi}^{2}=0\right)$

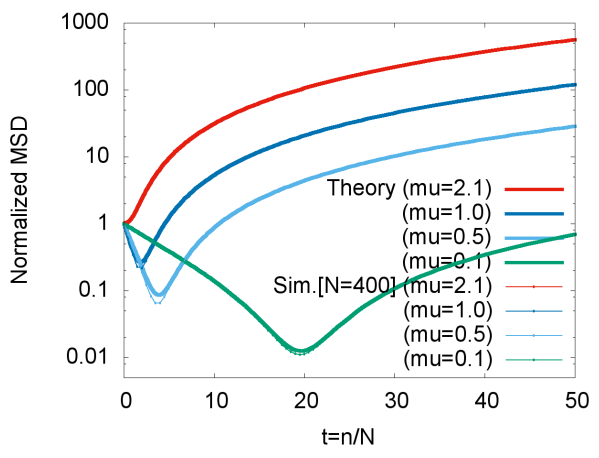

(a) $S=1$

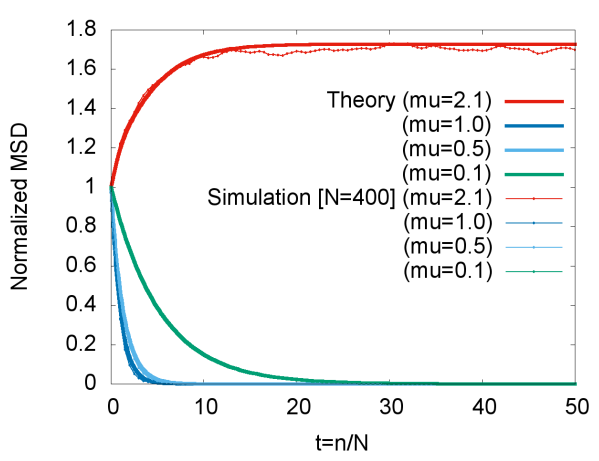

(b) $S=3$

Fig. 4: Normalized MSD learning curves $\left(\rho^{2}=\sigma_{g}^{2}=1, \sigma_{\xi}^{2}=0\right)$

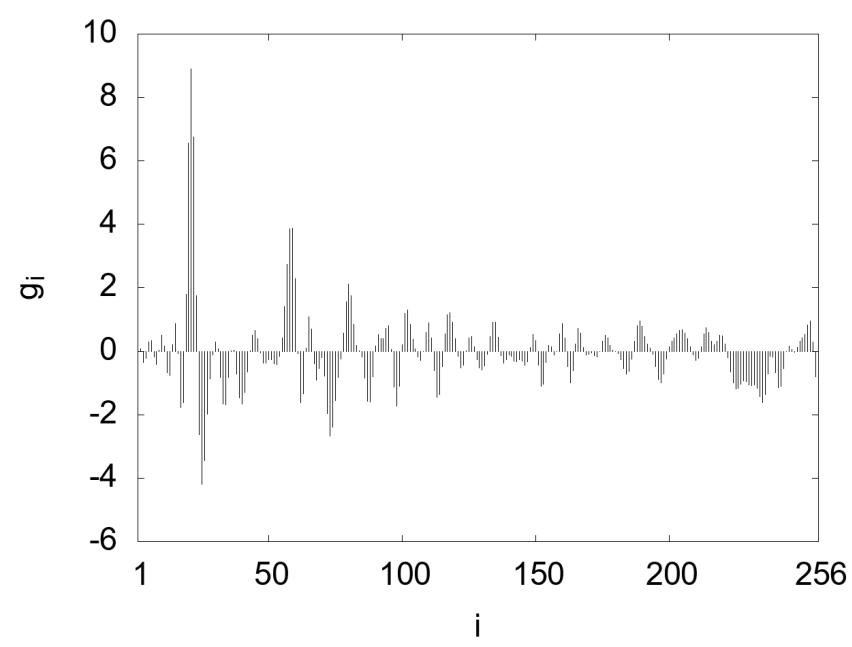

Fig. 5: Impulse response $\boldsymbol{g}_{0}$ of the unknown system $\mathrm{G}$ used in all computer simulations in this paper.

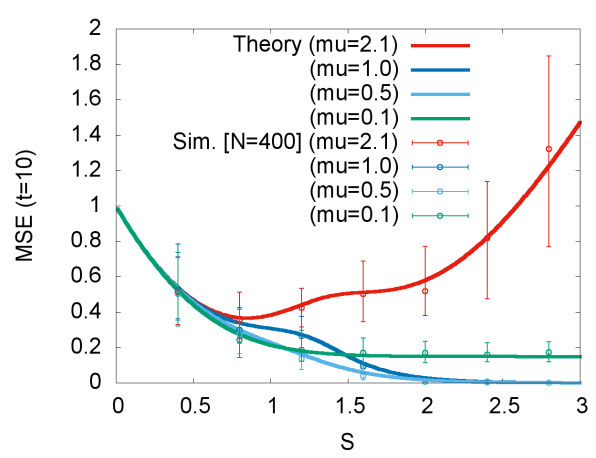

(a) $t=10$

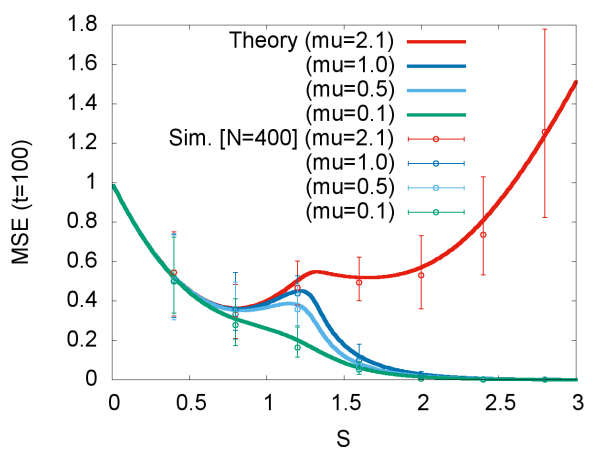

(b) $t=100$

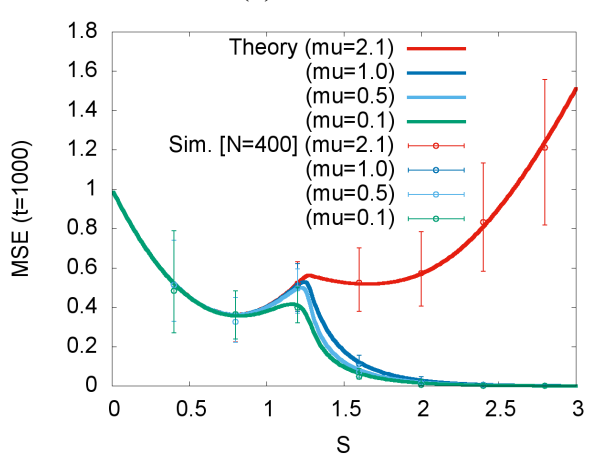

(c) $t=1000$

Fig. 6: $\operatorname{MSE}\left(\rho^{2}=\sigma_{g}^{2}=1, \sigma_{\xi}^{2}=0\right)$ 
values of the macroscopic variables $Q, r$, and MSE. If the steady-state values of $Q$ and $r$ exist, they can be obtained by numerically solving the simultaneous equations that are obtained by substituting zeros into the left-hand sides of the simultaneous differential equations (35) and (36). Figure 7 shows the results for $Q$. When the saturation value $S$ is larger than $S_{C}=1.25331 \cdots$, the solution is found. However, when $S$ is smaller than $S_{C}$, no solution is found. In addition, $Q$ diverges in the limit when $S$ approaches $S_{C}+0$. Since $Q$ is proportional to the square of the $\ell_{2}$-norm of $\boldsymbol{w}$ as seen from (18), the divergence of $Q$ means the divergence of the coefficient vector $\boldsymbol{w}$ of the adaptive filter W. When $S>S_{C}$, we can obtain the steady-state MSE by substituting the steadystate values of $Q$ and $r$ into (24). Note that it will become clear that the exact value of $S_{C}=1.25331 \cdots$ is $\sqrt{\frac{\pi}{2}}$ as described in Sec. IV-D.

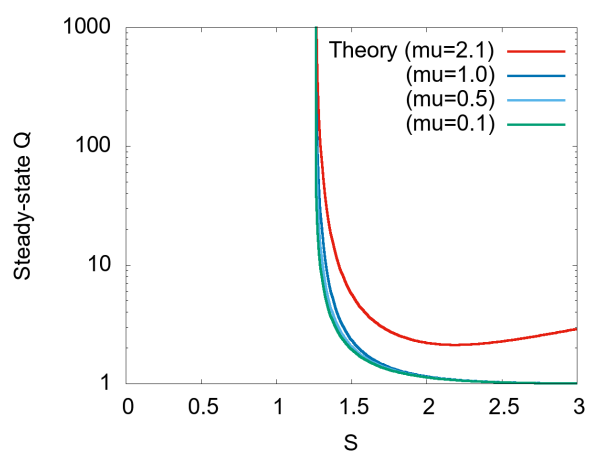

Fig. 7: Steady-state $Q\left(\rho^{2}=\sigma_{g}^{2}=1, \sigma_{\xi}^{2}=0\right)$

\section{Asymptotic analysis when $S<S_{C}$}

As described in the previous subsection, $Q$ diverges in the limit $t \rightarrow \infty$ when $S<S_{C}$. However, Fig. 6 shows that the MSE seems to converge even when $S<S_{C}$. Therefore, the asymptotic analysis of the behavior of the system when $S<S_{C}$ is shown in this subsection. From (6), (18), and (19), we obtain

$$
r=\sigma_{g} \sqrt{Q} \cos \theta .
$$

Here, $\theta$ is the angle between vectors $\boldsymbol{g}$ and $\boldsymbol{w}$. As described in Sec. IV-B, when $S<S_{C}, Q \rightarrow \infty$ in the limit $t \rightarrow \infty$. Therefore, from (35), (36), and (40), we obtain

$$
\cos \theta \stackrel{t \rightarrow \infty}{\longrightarrow} 1 \text {. }
$$

Equation (41) is derived in detail in Appendix E, where the approximations

$$
\begin{array}{rlrl}
\operatorname{erf}(x) & =\frac{2}{\sqrt{\pi}} \sum_{n=0}^{\infty} \frac{(-1)^{n} x^{2 n+1}}{n !(2 n+1)} \simeq \frac{2}{\sqrt{\pi}} x & & (|x| \ll 1), \\
\exp (x) & =\sum_{n=0}^{\infty} \frac{x^{n}}{n !} \simeq 1+x & (|x| \ll 1),
\end{array}
$$

are used.
Equation (41) means that the directions of $\boldsymbol{g}$ and $\boldsymbol{w}$ coincide even when $S<S_{C}$, although $\boldsymbol{w}$ diverges as described in Sec. IV-B. Note that this property does not depend on $\mu, \rho^{2}, \sigma_{g}^{2}$, nor $\sigma_{\xi}^{2}$.

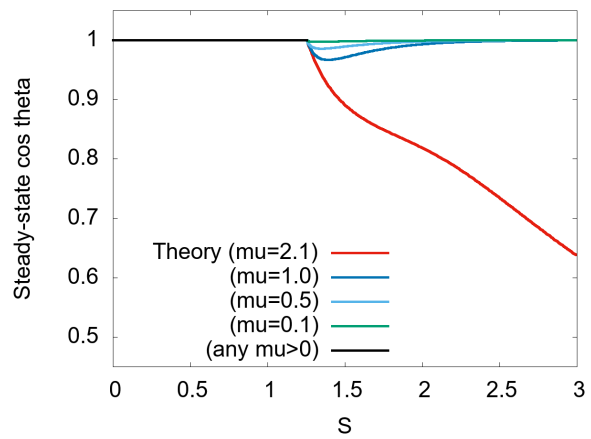

Fig. 8: Steady-state $\cos \theta=r /\left(\sigma_{g} \sqrt{Q}\right)\left(\rho^{2}=\sigma_{g}^{2}=1, \sigma_{\xi}^{2}=0\right)$

As described so far, $Q$ diverges and $\cos \theta=1$ in the limit $t \rightarrow \infty$ when $S<S_{C}$. Then, the MSE is

$$
\left\langle e^{2}\right\rangle=S^{2}-2 \sigma_{g} \rho \sqrt{\frac{2}{\pi}} S+\sigma_{g}^{2} \rho^{2}+\sigma_{\xi}^{2}
$$

Equation (44) is derived in detail in Appendix F. Equation (44) shows that the MSE converges although $\boldsymbol{w}$ diverges when $S<S_{C}$. The converged value does not depend on the step size $\mu$, and it is a quadratic function of $S$. The converged value is minimum, $\sigma_{g}^{2} \rho^{2}\left(1-\frac{2}{\pi}\right)+\sigma_{\xi}^{2}$, when $S=\sigma_{g} \rho \sqrt{\frac{2}{\pi}}$. Figure 8 shows the steady-state values of $\cos \theta=r /\left(\sigma_{g} \sqrt{Q}\right)$. In this figure, for $S>S_{C}$, values calculated using the steady-state values of $Q$ and $r$ that were given in Sec. IV-B are plotted. On the other hand, for $S<S_{C}$, the value obtained using (41) is plotted.

Figure 9 shows the steady-state values of the MSE. In this figure, for $S>S_{C}$, results of the steady-state analysis described in Sec. IV-B are plotted. The results show that the steady-state MSE depends on $\mu$. On the other hand, for $S<S_{C}$, the result obtained using (44) is plotted.

Figure 10 shows the steady-state normalized MSD. This figure shows that the normalized MSD diverges because $Q$ diverges in the limit $S \rightarrow S_{C}+0$. That is, the condition in which the adaptive system is mean-square stable [2] is $S>$ $S_{C}$.

\section{Exact derivation of the critical value $S_{C}$}

As described so far, the properties of the adaptive system switch markedly at $S=S_{C}$. That is, when $S>S_{C}$, the MSE and normalized MSD converge. That is, the adaptive system is mean-square stable. Converged values depend on the stepsize $\mu$. On the other hand, when $S<S_{C}$, the normalized MSD diverges and the MSE converges to the values that do not depend on $\mu$. In addition, the angle between the coefficient vector $\boldsymbol{g}$ of the unknown system and the coefficient vector $\boldsymbol{w}$ of the adaptive filter converges to zero. 


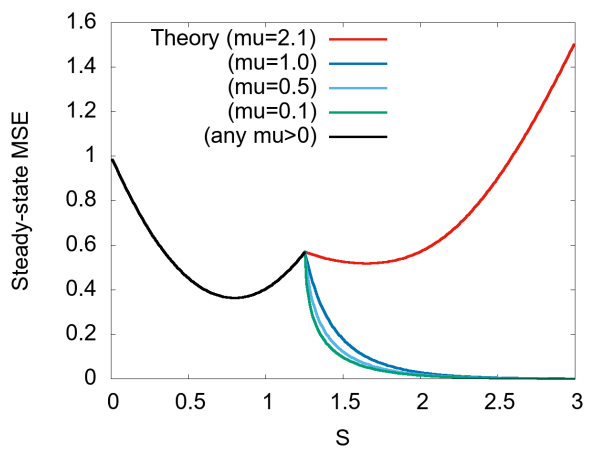

(a) $\sigma_{\xi}^{2}=0$

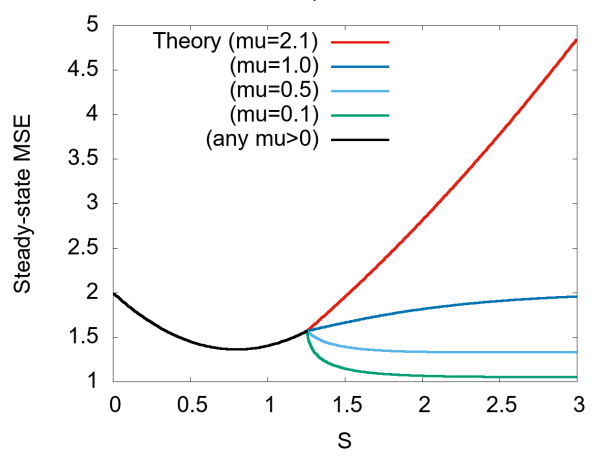

(b) $\sigma_{\xi}^{2}=1$

Fig. 9: Steady-state $\operatorname{MSE}\left(\rho^{2}=\sigma_{g}^{2}=1\right)$

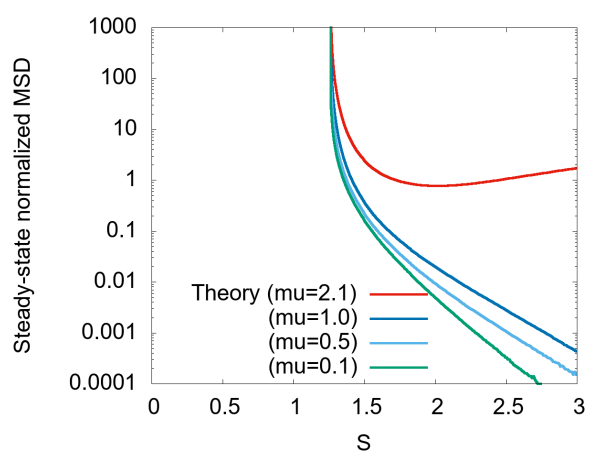

(a) $\sigma_{\xi}^{2}=0$

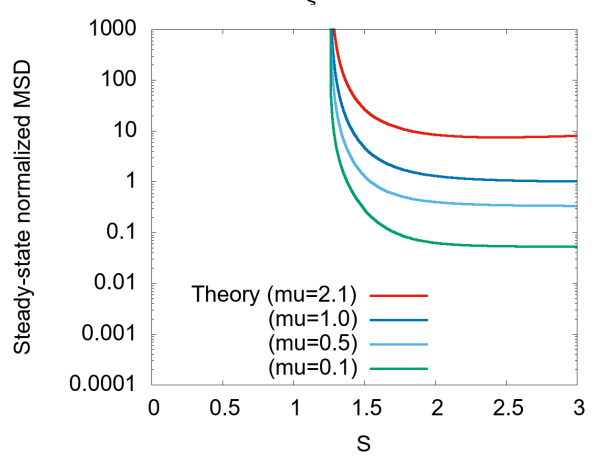

(b) $\sigma_{\xi}^{2}=1$

Fig. 10: Steady-state normalized $\operatorname{MSD}\left(\rho^{2}=\sigma_{g}^{2}=1\right)$
In this subsection, we analytically obtain the critical value $S_{C}$. As described in Sec. IV-B,

$$
\lim _{S \rightarrow S_{C}+0} \lim _{t \rightarrow \infty} \frac{\mathrm{d} r}{\mathrm{~d} t}=\lim _{S \rightarrow S_{C}+0} \lim _{t \rightarrow \infty} \frac{\mathrm{d} Q}{\mathrm{~d} t}=0 .
$$

As described in Secs. IV-B and IV-C,

$$
\begin{aligned}
\lim _{S \rightarrow S_{C}+0} \lim _{t \rightarrow \infty} Q & =\infty, \\
\lim _{S \rightarrow S_{C}+0} \lim _{t \rightarrow \infty} \cos \theta & =1 .
\end{aligned}
$$

Substituting (42), (43), and (45)-(47) into (35) and (36) and solving for $S_{C}$, we obtain

$$
S_{C}=\sigma_{g} \rho \sqrt{\frac{\pi}{2}} .
$$

Equation (48) shows that the critical value $S_{C}$ is proportional to $\sigma_{g}$ and $\rho$ defined by (16) and (6), respectively. In addition, we see that the critical value of $S_{C}=1.25331 \cdots$ numerically obtained in Sec. IV-B is, in fact, $\sqrt{\frac{\pi}{2}}$.

\section{Conclusions}

We have analyzed the behaviors of the adaptive system in which the output of the adaptive filter has the clipping saturation-type nonlinearity by the statistical-mechanical method. To represent the macroscopic state of the system, we have introduced two macroscopic variables $Q$ and $r$. By considering the limit in which the number of taps of the adaptive filter is large, we have derived the simultaneous differential equations that describe the system behaviors in the deterministic and closed form. Although the derived equations cannot be analytically solved, we have discussed the dynamical behaviors and steady state of the adaptive system by numerical calculation, asymptotic analysis, and steady-state analysis. As a result, it has become clear that the saturation value $S$ has the critical value $S_{C}$ at which the system's meansquare stability and instability switch. That is, when $S>S_{C}$, both the mean-square error (MSE) and mean-square deviation (MSD) converge, i.e., the adaptive system is mean-square stable. On the other hand, when $S<S_{C}$, the MSD diverges although the MSE converges, i.e., the adaptive system is not mean-square stable. In the latter case, the converged value of the MSE is a quadratic function of $S$ and does not depend on the step size. Finally, $S_{C}$ has been exactly derived by asymptotic analysis. The findings in this study are non-trivial, albeit with respect to classical and simple models, and are significant both theoretically and practically.

There remain interesting theoretical problems that should be studied further. For example, there are analyses of other types of nonlinearities. In this paper, we have analyzed the clipping saturation-type nonlinearity. As described before, this is the most direct expression of the saturation characteristics of power amplifiers and transducers such as loudspeakers and microphones. On the other hand, there are indeed many other types of nonlinearity. For example, Costa et al. [27] analyzed a dead-zone-type nonlinearity. As described before, the deadzone-type nonlinearity is a model of the characteristics of class B amplifiers and nonlinear actuators. Just as most of the previous studies on saturation characteristics analyzed smooth 
nonlinearity expressed by erf-type function, Costa et al. [27] approximated a dead-zone-type nonlinearity with an erf-type function. As with the saturation nonlinearity analyzed in this paper, a dead-zone-type nonlinearity can be expressed more directly, that is, by a piecewise linearity. In that case, the analysis can be performed by the method described in this paper. The nonlinearities of the other components are also issues for future studies. In this paper, we analyzed a model in which the output of the adaptive filter has nonlinearity. As described before, this is a model of, for example, ANC. On the other hand, a model with saturation nonlinearities in the output of the unknown system is a model for an echo canceller. Such a model can be analyzed by the method described in this paper. The same analysis can be performed for the case where the error signal undergoes a nonlinear transformation. As discovered in this study that there is a critical point in the saturation value, the existence of as yet undiscovered phenomena may become apparent in these analyses. Thus, these analyses are interesting tasks in the near future.

\section{ACKNOWLEDGEMENTS}

The author wishes to thank Professor Yoshinobu Kajikawa for providing the actual data of the experimentally measured impulse response. 


\section{APPENDIX A}

\section{DERIVATION OF MEANS AND VARIANCE-COVARIANCE MATRIX OF $d$ AND $y$}

From (6), (7), (9), (10), (16), (18), and (19), we obtain means, variances, and covariance of $d$ and $y$ as follows:

$$
\begin{aligned}
\langle d\rangle & =\left\langle\sum_{i=1}^{N} g_{i} u(n-i+1)\right\rangle=\sum_{i=1}^{N} g_{i}\langle u(n-i+1)\rangle=0 \\
\langle y\rangle & =\left\langle\sum_{i=1}^{N} w_{i} u(n-i+1)\right\rangle=\sum_{i=1}^{N} w_{i}\langle u(n-i+1)\rangle=0 \\
\left\langle d^{2}\right\rangle & =\left\langle\left(\sum_{i=1}^{N} g_{i} u(n-i+1)\right)^{2}\right\rangle=\left\langle\sum_{i=1}^{N} \sum_{j=1}^{N} g_{i} g_{j} u(n-i+1) u(n-j+1)\right\rangle \\
& =\sum_{i=1}^{N} g_{i}^{2}\left\langle u(n-i+1)^{2}\right\rangle=\sigma^{2} \sum_{i=1}^{N} g_{i}^{2} \stackrel{N \rightarrow \infty}{\longrightarrow} \rho^{2} \sigma_{g}^{2}, \\
\left\langle y^{2}\right\rangle & =\left\langle\left(\sum_{i=1}^{N} w_{i} u(n-i+1)\right)^{2}\right\rangle=\left\langle\sum_{i=1}^{N} \sum_{j=1}^{N} w_{i} w_{j} u(n-i+1) u(n-j+1)\right\rangle \\
& =\sum_{i=1}^{N} w_{i}^{2}\left\langle u(n-i+1)^{2}\right\rangle=\sigma^{2} \sum_{i=1}^{N} w_{i}^{2} \stackrel{N \rightarrow \infty}{\longrightarrow} \rho^{2} Q, \\
\langle d y\rangle & =\left\langle\left(\sum_{i=1}^{N} g_{i} u(n-i+1)\right)\left(\sum_{j=1}^{N} w_{j} u(n-j+1)\right)\right\rangle=\left\langle\sum_{i=1}^{N} \sum_{j=1}^{N} g_{i} w_{j} u(n-i+1) u(n-j+1)\right\rangle \\
& =\sum_{i=1}^{N} g_{i} w_{i}\left\langle u(n-i+1)^{2}\right\rangle=\sigma^{2} \sum_{i=1}^{N} g_{i} w_{i} \stackrel{N \rightarrow \infty}{\longrightarrow} \rho^{2} r .
\end{aligned}
$$

From (49)-(53), the covariance matrix of $d$ and $y$ is (17). Here, (50), (52), and (53) were derived assuming that the correlation between $\boldsymbol{w}(n)$ and $\boldsymbol{u}(n)$ is small [24], [40], [41]. This assumption is a standard assumption used to analyze many adaptive algorithms [1], [2].

\section{APPENDIX B}

DERIVATION OF (21)

$$
\begin{aligned}
\left\langle f(y)^{2}\right\rangle & =\int_{-\infty}^{\infty} \mathrm{d} y f(y)^{2} p(y)=\left(\int_{-\infty}^{-S}+\int_{-S}^{S}+\int_{S}^{\infty}\right) \mathrm{d} y f(y)^{2} \frac{1}{\sqrt{2 \pi \rho^{2} Q}} \exp \left(-\frac{y^{2}}{2 \rho^{2} Q}\right) \\
& =2(\underbrace{\int_{S}^{\infty} \mathrm{d} y S^{2} \frac{1}{\sqrt{2 \pi \rho^{2} Q}} \exp \left(-\frac{y^{2}}{2 \rho^{2} Q}\right)}_{B 1}+\underbrace{\int_{0}^{S} \mathrm{~d} y y^{2} \frac{1}{\sqrt{2 \pi \rho^{2} Q}} \exp \left(-\frac{y^{2}}{2 \rho^{2} Q}\right)}_{B 2}),
\end{aligned}
$$

$$
\begin{array}{rlr}
B 1 & =\int_{S}^{\infty} \mathrm{d} y S^{2} \frac{1}{\sqrt{2 \pi \rho^{2} Q}} \exp \left(-\frac{y^{2}}{2 \rho^{2} Q}\right)=\frac{S^{2}}{\sqrt{\pi}} \int_{\frac{S}{\sqrt{2 \rho^{2} Q}}}^{\infty} \mathrm{d} y^{\prime} \exp \left(-y^{\prime 2}\right), & \text { where } y^{\prime}=\frac{y}{\sqrt{2 \rho^{2} Q}} \\
& =\frac{S^{2}}{\sqrt{\pi}}\left(\frac{\sqrt{\pi}}{2}-\int_{0}^{\frac{S}{\sqrt{2 \rho^{2} Q}}} \mathrm{~d} y \exp \left(-y^{2}\right)\right) & \left(\because \int_{0}^{\infty} \mathrm{d} y \exp \left(-y^{2}\right)=\frac{\sqrt{\pi}}{2}\right) \\
& =\frac{S^{2}}{2}\left(1-\operatorname{erf}\left(\frac{S}{\sqrt{2 \rho^{2} Q}}\right)\right),
\end{array}
$$




$$
\begin{aligned}
B 2 & =\int_{0}^{S} \mathrm{~d} y y^{2} \frac{1}{\sqrt{2 \pi \rho^{2} Q}} \exp \left(-\frac{y^{2}}{2 \rho^{2} Q}\right) \\
& =\left[-y \sqrt{\frac{\rho^{2} Q}{2 \pi}} \exp \left(-\frac{y^{2}}{2 \rho^{2} Q}\right)\right]_{0}^{S}+\sqrt{\frac{\rho^{2} Q}{2 \pi}} \int_{0}^{S} \mathrm{~d} y \exp \left(-\frac{y^{2}}{2 \rho^{2} Q}\right), \quad \text { where we used integration by parts } \\
& =-S \sqrt{\frac{\rho^{2} Q}{2 \pi}} \exp \left(-\frac{S^{2}}{2 \rho^{2} Q}\right)+\frac{\rho^{2} Q}{2} \int_{0}^{\frac{S}{\sqrt{\rho^{2} Q}}} \mathrm{~d} y^{\prime} \exp \left(-y^{\prime 2}\right), \quad \text { where } y^{\prime}=\frac{y}{\sqrt{2 \rho^{2} Q}} \\
& =-S \sqrt{\frac{\rho^{2} Q}{2 \pi}} \exp \left(-\frac{S^{2}}{2 \rho^{2} Q}\right)+\frac{\rho^{2} Q}{2} \operatorname{erf}\left(\frac{S}{\sqrt{2 \rho^{2} Q}}\right), \\
\therefore\left\langle f(y)^{2}\right\rangle & =2(B 1+B 2)=S^{2}-S \sqrt{\frac{\rho^{2} Q}{2 \pi}} \exp \left(-\frac{S^{2}}{2 \rho^{2} Q}\right)+\left(\rho^{2} Q-S^{2}\right) \operatorname{erf}\left(\frac{S}{\sqrt{2 \rho^{2} Q}}\right) .
\end{aligned}
$$

\section{APPENDIX C}

DERIVATION OF (22)

$$
\begin{aligned}
& \langle d f(y)\rangle=\int_{-\infty}^{\infty} \int_{-\infty}^{\infty} \mathrm{d} d \mathrm{~d} y d f(y) p(d, y) \\
& =\underbrace{\int_{-\infty}^{\infty} \mathrm{d} d d \int_{-\infty}^{-S} \mathrm{~d} y(-S) p(d, y)}_{C 1}+\underbrace{\int_{-\infty}^{\infty} \mathrm{d} d d \int_{-S}^{S} \mathrm{~d} y y p(d, y)}_{C 2}+\underbrace{\int_{-\infty}^{\infty} \mathrm{d} d d \int_{S}^{\infty} \mathrm{d} y S p(d, y)}_{C 3}, \\
& C 2=\int_{-\infty}^{\infty} \mathrm{d} d d \int_{-S}^{S} \mathrm{~d} y y \frac{1}{2 \pi \sqrt{\left|\rho^{2}\left(\begin{array}{cc}
\sigma_{g}^{2} & r \\
r & Q
\end{array}\right)\right|}} \exp \left(-\frac{\left(\begin{array}{ll}
d & y
\end{array}\right)\left(\rho^{2}\left(\begin{array}{cc}
\sigma_{g}^{2} & r \\
r & Q
\end{array}\right)\right)^{-1}\left(\begin{array}{l}
d \\
y
\end{array}\right)}{2}\right) \\
& =\int_{-\infty}^{\infty} \mathrm{d} d d \int_{-S}^{S} \mathrm{~d} y y \frac{1}{2 \pi \rho^{2} \sqrt{\sigma_{g}^{2} Q-r^{2}}} \exp \left(-\frac{Q d^{2}-2 r d y+\sigma_{g}^{2} y^{2}}{2 \rho^{2}\left(\sigma_{g}^{2} Q-r^{2}\right)}\right) \\
& =\int_{-\infty}^{\infty} \mathrm{d} d d \int_{-S}^{S} \mathrm{~d} y y \frac{1}{2 \pi \rho^{2} \sqrt{\sigma_{g}^{2} Q-r^{2}}} \exp \left(-\frac{Q\left(d-\frac{r}{Q} y\right)^{2}+\left(\sigma_{g}^{2}-\frac{r^{2}}{Q}\right) y^{2}}{2 \rho^{2}\left(\sigma_{g}^{2} Q-r^{2}\right)}\right) \\
& =\int_{-S}^{S} \mathrm{~d} y y \exp \left(-\frac{y^{2}}{2 \rho^{2} Q}\right) \int_{-\infty}^{\infty} \mathrm{d} d d \frac{1}{2 \pi \rho^{2} \sqrt{\sigma_{g}^{2} Q-r^{2}}} \exp \left(-\frac{\left(d-\frac{r}{Q} y\right)^{2}}{2 \rho^{2}\left(\sigma_{g}^{2}-\frac{r^{2}}{Q}\right)}\right) \\
& =\int_{-S}^{S} \mathrm{~d} y y \exp \left(-\frac{y^{2}}{2 \rho^{2} Q}\right) \int_{-\infty}^{\infty} \sqrt{2 \rho^{2}\left(\sigma_{g}^{2}-\frac{r^{2}}{Q}\right)} \mathrm{d} d^{\prime}\left(\sqrt{2 \rho^{2}\left(\sigma_{g}^{2}-\frac{r^{2}}{Q}\right)} d^{\prime}+\frac{r}{Q} y\right) \frac{1}{2 \pi \rho^{2} \sqrt{\sigma_{g}^{2} Q-r^{2}}} \exp \left(-d^{\prime 2}\right), \\
& \text { where } d^{\prime}=\frac{d-\frac{r}{Q} y}{\sqrt{2 \rho^{2}\left(\sigma_{g}^{2}-\frac{r^{2}}{Q}\right)}} \\
& =\int_{-S}^{S} \mathrm{~d} y y \exp \left(-\frac{y^{2}}{2 \rho^{2} Q}\right) \int_{-\infty}^{\infty} 2 \rho^{2}\left(\sigma_{g}^{2}-\frac{r^{2}}{Q}\right) \mathrm{d} d d \frac{1}{2 \pi \rho^{2} \sqrt{\sigma_{g}^{2} Q-r^{2}}} \exp \left(-d^{2}\right) \\
& +\int_{-S}^{S} \frac{r}{Q} \mathrm{~d} y y^{2} \exp \left(-\frac{y^{2}}{2 \rho^{2} Q}\right) \int_{-\infty}^{\infty} \sqrt{2 \rho^{2}\left(\sigma_{g}^{2}-\frac{r^{2}}{Q}\right)} \mathrm{d} d \frac{1}{2 \pi \rho^{2} \sqrt{\sigma_{g}^{2} Q-r^{2}}} \exp \left(-d^{2}\right)
\end{aligned}
$$


STATISTICAL-MECHANICAL ANALYSIS OF ADAPTIVE FILTER WITH CLIPPING SATURATION-TYPE NONLINEARITY

11

$$
\begin{aligned}
& =\int_{-S}^{S} \frac{r}{Q} \mathrm{~d} y y^{2} \exp \left(-\frac{y^{2}}{2 \rho^{2} Q}\right) \int_{-\infty}^{\infty} \mathrm{d} d \frac{1}{\pi \sqrt{2 \rho^{2} Q}} \exp \left(-d^{2}\right) \quad\left(\because \int_{-S}^{S} \mathrm{~d} y y \exp \left(-\frac{y^{2}}{2 \rho^{2} Q}\right)=0\right) \\
& =\frac{2 r}{Q \sqrt{2 \pi \rho^{2} Q}} \int_{0}^{S} \mathrm{~d} y y^{2} \exp \left(-\frac{y^{2}}{2 \rho^{2} Q}\right) \\
& =\frac{2 r}{Q \sqrt{2 \pi \rho^{2} Q}}\left(\left[-\rho^{2} Q y \exp \left(-\frac{y^{2}}{2 \rho^{2} Q}\right)\right]_{0}^{\infty}-\int_{0}^{S} \mathrm{~d} d \exp \left(-d^{2}\right)=\sqrt{\pi}\right)
\end{aligned}
$$

where we used integration by parts

$$
\begin{aligned}
& =\frac{2 r}{Q \sqrt{2 \pi \rho^{2} Q}}\left(-\rho^{2} S Q \exp \left(-\frac{S^{2}}{2 \rho^{2} Q}\right)+\rho^{2} Q \int_{0}^{\frac{S}{\sqrt{2 \rho^{2} Q}}} \sqrt{2 \rho^{2} Q} \mathrm{~d} y^{\prime} \exp \left(-y^{\prime 2}\right)\right), \quad \text { where } y^{\prime}=\frac{y}{\sqrt{2 \rho^{2} Q}} \\
& =-r S \rho \sqrt{\frac{2}{\pi Q}} \exp \left(-\frac{S^{2}}{2 \rho^{2} Q}\right)+\rho^{2} r \operatorname{erf}\left(\frac{S}{\sqrt{2 \rho^{2} Q}}\right),
\end{aligned}
$$

$$
\begin{aligned}
& \begin{aligned}
C 3 & =\int_{-\infty}^{\infty} \mathrm{d} d d \int_{S}^{\infty} \mathrm{d} y S \frac{1}{2 \pi \sqrt{\left|\rho^{2}\left(\begin{array}{cc}
\sigma_{g}^{2} & r \\
r & Q
\end{array}\right)\right|}} \exp \left(-\frac{\left(\begin{array}{ll}
d & y
\end{array}\right)\left(\rho^{2}\left(\begin{array}{cc}
\sigma_{g}^{2} & r \\
r & Q
\end{array}\right)\right)^{-1}\left(\begin{array}{l}
d \\
y
\end{array}\right)}{2}\right) \\
& =\int_{-\infty}^{\infty} \mathrm{d} d d \int_{S}^{\infty} \mathrm{d} y S \frac{1}{2 \pi \rho^{2} \sqrt{\sigma_{g}^{2} Q-r^{2}}} \exp \left(-\frac{Q d^{2}-2 r d y+\sigma_{g}^{2} y^{2}}{2 \rho^{2}\left(\sigma_{g}^{2} Q-r^{2}\right)}\right) \\
& =\int_{-\infty}^{\infty} \mathrm{d} d d \int_{S}^{\infty} \mathrm{d} y S \frac{1}{2 \pi \rho^{2} \sqrt{\sigma_{g}^{2} Q-r^{2}}} \exp \left(-\frac{Q\left(d-\frac{r}{Q} y\right)^{2}+\left(\sigma_{g}^{2}-\frac{r^{2}}{Q}\right) y^{2}}{2 \rho^{2}\left(\sigma_{g}^{2} Q-r^{2}\right)}\right) \\
& =S \int_{S}^{\infty} \mathrm{d} y \exp \left(-\frac{y^{2}}{2 \rho^{2} Q}\right) \int_{-\infty}^{\infty} \mathrm{d} d d \frac{1}{2 \pi \rho^{2} \sqrt{\sigma_{g}^{2} Q-r^{2}}} \exp \left(-\frac{\left(d-\frac{r}{Q} y\right)^{2}}{2 \rho^{2}\left(\sigma_{g}^{2}-\frac{r^{2}}{Q}\right)}\right) \\
& =S \int_{S}^{\infty} \mathrm{d} y \exp \left(-\frac{y^{2}}{2 \rho^{2} Q}\right) \int_{-\infty}^{\infty} \sqrt{2 \rho^{2}\left(\sigma_{g}^{2}-\frac{r^{2}}{Q}\right)} \mathrm{d} d^{\prime}\left(\sqrt{2 \rho^{2}\left(\sigma_{g}^{2}-\frac{r^{2}}{Q}\right)} d^{\prime}+\frac{r}{Q} y\right) \frac{1}{2 \pi \rho^{2} \sqrt{\sigma_{g}^{2} Q-r^{2}}} \exp \left(-d^{\prime 2}\right)
\end{aligned} \\
& \text { where } d^{\prime}=\frac{d-\frac{r}{Q} y}{\sqrt{2 \rho^{2}\left(\sigma_{g}^{2}-\frac{r^{2}}{Q}\right)}} \\
& =S \int_{S}^{\infty} \mathrm{d} y \exp \left(-\frac{y^{2}}{2 \rho^{2} Q}\right) \int_{-\infty}^{\infty} 2 \rho^{2}\left(\sigma_{g}^{2}-\frac{r^{2}}{Q}\right) \mathrm{d} d d \frac{1}{2 \pi \rho^{2} \sqrt{\sigma_{g}^{2} Q-r^{2}}} \exp \left(-d^{2}\right) \\
& +S \int_{S}^{\infty} \frac{r}{Q} \mathrm{~d} y y \exp \left(-\frac{y^{2}}{2 \rho^{2} Q}\right) \int_{-\infty}^{\infty} \sqrt{2 \rho^{2}\left(\sigma_{g}^{2}-\frac{r^{2}}{Q}\right)} \mathrm{d} d \frac{1}{2 \pi \rho^{2} \sqrt{\sigma_{g}^{2} Q-r^{2}}} \exp \left(-d^{2}\right) \\
& =\frac{S r}{Q} \int_{S}^{\infty} \mathrm{d} y y \exp \left(-\frac{y^{2}}{2 \rho^{2} Q}\right) \sqrt{2 \rho^{2}\left(\sigma_{g}^{2}-\frac{r^{2}}{Q}\right)} \frac{1}{2 \pi \rho^{2} \sqrt{\sigma_{g}^{2} Q-r^{2}}} \sqrt{\pi} \\
& \left(\because \int_{-\infty}^{\infty} \mathrm{d} d d \exp \left(-d^{2}\right)=0, \quad \int_{-\infty}^{\infty} \mathrm{d} d \exp \left(-d^{2}\right)=\sqrt{\pi}\right) \\
& =\frac{S r}{Q} \frac{1}{\sqrt{2 \pi \rho^{2} Q}}\left[\left(-\rho^{2} Q\right) \exp \left(-\frac{y^{2}}{2 \rho^{2} Q}\right)\right]_{S}^{\infty}=\frac{S r \rho}{\sqrt{2 \pi Q}} \exp \left(-\frac{S^{2}}{2 \rho^{2} Q}\right) \text {, } \\
& C 1=\int_{-\infty}^{\infty} \mathrm{d} d d \int_{-\infty}^{-S} \mathrm{~d} y(-S) p(d, y)=C 3, \quad \text { where we used integration by substitution: } y^{\prime}=-y, d^{\prime}=d,
\end{aligned}
$$




$$
\therefore\langle d f(y)\rangle=C 1+C 2+C 3=\rho^{2} r \operatorname{erf}\left(\frac{S}{\sqrt{2 \rho^{2} Q}}\right) \text {. }
$$

APPENDIX D

DERIVATION OF (34)

$$
\begin{aligned}
& \langle y(f(y)\rangle=\int_{-\infty}^{\infty} \mathrm{d} y y f(y) p(y)=\underbrace{\int_{-\infty}^{-S} \mathrm{~d} y y(-S) p(y)}_{D 1}+\underbrace{\int_{-S}^{S} \mathrm{~d} y y^{2} p(y)}_{D 2}+\underbrace{\int_{S}^{\infty} \mathrm{d} y y S p(y)}_{D 3} \\
& D 2=-S \sqrt{\frac{2 \rho^{2} Q}{\pi}} \exp \left(-\frac{S^{2}}{2 \rho^{2} Q}\right)+\rho^{2} Q \operatorname{erf}\left(\frac{S}{\sqrt{2 \rho^{2} Q}}\right), \\
& D 1=D 3=S \int_{S}^{\infty} \mathrm{d} y y \frac{1}{\sqrt{2 \pi \rho^{2} Q}} \exp \left(-\frac{y^{2}}{2 \rho^{2} Q}\right)=\frac{S}{\sqrt{2 \rho^{2} Q}}\left[-\rho^{2} Q \exp \left(-\frac{y^{2}}{2 \rho^{2} Q}\right)\right]_{S}^{\infty}=S \sqrt{\frac{\rho^{2} Q}{2 \pi}} \exp \left(-\frac{S^{2}}{2 \rho^{2} Q}\right) . \\
& \therefore\langle y f(y)\rangle=D 1+D 2+D 3=\rho^{2} Q \operatorname{erf}\left(\frac{S}{\sqrt{2 \rho^{2} Q}}\right) .
\end{aligned}
$$

\section{APPENDIX E}

DERIVATION OF (41)

When $S<S_{C}$, since $Q \rightarrow \infty$ in the limit $t \rightarrow \infty$, from (35), (40), and (42), we obtain

$$
\begin{aligned}
\frac{\mathrm{d} r}{\mathrm{~d} t} & =\sigma_{g} \cos \theta \frac{d \sqrt{Q}}{\mathrm{~d} t}=\mu \rho^{2}\left(\sigma_{g}^{2}-\sigma_{g} \cos \theta \sqrt{Q} \operatorname{erf}\left(\frac{S}{\sqrt{2 \rho^{2} Q}}\right)\right) \simeq \mu \rho^{2}\left(\sigma_{g}^{2}-\sigma_{g} \cos \theta \sqrt{Q} \frac{2}{\sqrt{\pi}} \frac{S}{\sqrt{2 \rho^{2} Q}}\right), \\
\therefore \frac{d \sqrt{Q}}{\mathrm{~d} t} & \simeq \mu \rho^{2}\left(\frac{\sigma_{g}}{\cos \theta}-\sqrt{\frac{2}{\pi \rho^{2}}} S\right) .
\end{aligned}
$$

On the other hand, from (36), (40), (42), and (43), we obtain

$$
\begin{aligned}
\frac{\mathrm{d} Q}{\mathrm{~d} t}= & \frac{\mathrm{d}(\sqrt{Q})^{2}}{\mathrm{~d} t}=2 \sqrt{Q} \frac{d \sqrt{Q}}{\mathrm{~d} t} \\
\simeq & \mu \rho^{2}\left(\mu\left(\rho^{2} Q-2 \rho^{2} \sigma_{g} \sqrt{Q} \cos \theta-S^{2}\right)-2 Q\right) \frac{2}{\sqrt{\pi}} \frac{S}{\sqrt{2 \rho^{2} Q}}-\mu^{2} \rho^{2} S \sqrt{\frac{2 \rho^{2} Q}{\pi}}\left(1-\frac{S^{2}}{2 \rho^{2} Q}\right) \\
& +\mu \rho^{2}\left(\mu\left(\rho^{2} \sigma_{g}^{2}+S^{2}+\sigma_{\xi}^{2}\right)+2 \sigma_{g} \sqrt{Q} \cos \theta\right) .
\end{aligned}
$$

The right-hand side of the formula that is obtained by dividing both sides of (97) by $2 \sqrt{Q}$ should be equal to the right-hand side of (95). Therefore, solving the equation for $\cos \theta$, we obtain

$$
\cos \theta \stackrel{t \rightarrow \infty}{\longrightarrow} 1 \text {. }
$$

\section{APPENDIX F}

DERIVATION OF (44)

When $S<S_{C}, Q \rightarrow \infty$ and $\cos \theta=1$ in the limit $t \rightarrow \infty$. Therefore, from (95), we obtain

$$
\begin{aligned}
& \frac{\mathrm{d} \sqrt{Q}}{\mathrm{~d} t} \simeq \mu \rho^{2}\left(\sigma_{g}-\sqrt{\frac{2}{\pi \rho^{2}}} S\right), \\
\therefore \sqrt{Q} & \simeq \mu \rho^{2}\left(\sigma_{g}-\sqrt{\frac{2}{\pi \rho^{2}}} S\right) t+\text { Const.. }
\end{aligned}
$$

On the other hand, from (40), we obtain

$$
r \simeq \sigma_{g} \sqrt{Q} .
$$

By substituting (42), (43), (100), and (101) into (24) and arranging the expression, we obtain the MSE as

$$
\left\langle e^{2}\right\rangle=S^{2}-2 \sigma_{g} \rho \sqrt{\frac{2}{\pi}} S+\sigma_{g}^{2} \rho^{2}+\sigma_{\xi}^{2}
$$




\section{REFERENCES}

[1] S. Haykin, Adaptive Filter Theory, $4^{\text {th }}$ ed., Prentice Hall, Upper Saddle River, NJ, 2002.

[2] A. H. Sayed, Fundamentals of Adaptive Filtering, Wiley, Hoboken, NJ, 2003.

[3] P. A. Nelson and S. J. Elliott, Active Control of Sound, Academic Press, San Diego, CA, 1992.

[4] S. M. Kuo and D. R. Morgan, Active Noise Control Systems Algorithms and DSP Implementations, Wiley, New York, 1996.

[5] S. M. Kuo and D. R. Morgan, "Active noise control: a tutorial review," Proc. IEEE, vol. 87, no. 6, pp. 943-973, June 1999.

[6] Y. Kajikawa, W.-S. Gan, and S. M. Kuo, "Recent advances on active noise control: Open issues and innovative applications," APSIPA Trans. Signal Inf. Process., vol. 1, e3, Aug. 2012.

[7] C. R. Fuller and A. H. von Flotow, "Active control of sound and vibration," IEEE Contr. Syst. Mag., vol. 15, no. 6, Dec. 1995.

[8] M. M. Sondhi, "The history of echo cancellation," IEEE Signal Process. Mag. vol. 23, no. 5, Sept. 2006.

[9] L. Ljung, System Identification: Theory for the User, $2^{\text {nd }}$ ed., Prentice Hall, Upper Saddle River, NJ, 1999.

[10] B. Widrow, J. L. Moschner, and J. Kaunitz, "Effects of quantization in adaptive processes. A hybrid adaptive processor," Stanford Electron Lab., Rep. 6793-1, 1971.

[11] T. A. C. M. Claasen and W. F. G. Mecklenbräuker, "Comparison of the convergence of two algorithms for adaptive FIR digital filters," IEEE Trans. Acoust. Speech, Signal Process., vol. 29, no. 3, pp. 670-678, 1981.

[12] D. L. Duttweiler, "Adaptive filter performance with nonlinearities in the correlation multiplier," IEEE Trans. Acoust. Speech, Signal Process., vol 30 , no. 4 , pp. $578-586,1982$.

[13] A. Aref and M. Lotfizad, "Variable step size modified clipped LMS algorithm," Proc. 2nd Int. Conf. Knowledge-Based Engineering and Innovation (KBEI), pp. 546-550, 2015.

[14] M. K. Smaoui, Y. B. Jemaa, and M. Jaidane, "How does the clipped LMS outperform the LMS?," Proc. 19th European Signal Processing Conference (EUSIPCO), pp. 734-738, 2011.

[15] M. Bekrani and A. W. H. Khong, "Misalignment analysis and insights into the performance of clipped-input LMS with correlated Gaussian data," Proc. IEEE Int. Conf. Acoustics, Speech, and Signal Processing (ICASSP), pp. 5929-5933, 2014.

[16] L. Deivasigamani, "A fast clipped-data LMS algorithm," IEEE Trans. Acoust. Speech, Signal Process., vol. 30, no. 4, pp. 648-649, 1982.

[17] M. Bekrani and A. W. H. Khong, "Convergence analysis of clipped input adaptive filters applied to system identification," Proc. Forty Sixth Asilomar Conference on Signals, Systems and Computers (ASILOMAR), pp. 801-805, 2012.

[18] B. E. Jun, D. J. Park, and Y. W. Kim, "Convergence analysis of sign-sign LMS algorithm for adaptive filters with correlated Gaussian data," Proc. IEEE Int. Conf. Acoustics, Speech, and Signal Processing (ICASSP), pp. 1380-1383, 1995

[19] K. Takahashi and S. Mori, "A new normalized signed regressor LMS algorithm," Proc. Singapore ICCS/ISITA, pp. 1181-1185, 1992.

[20] E. Eweda, "Analysis and design of a signed regressor LMS algorithm for stationary and nonstationary adaptive filtering with correlated Gaussian data," IEEE Trans. Circuits Syst., vol. 37, no. 11, pp. 1367-1374, 1990.

[21] N. J. Bershad, "On weight update saturation nonlinearities in LMS adaptation," IEEE Trans. Acoust. Speech, Signal Process., vol. 38, no. 4, pp. 623-630, 1990.

[22] M. H. Costa, J. C. M. Bermudez, and N. J. Bershad, "Statistical analysis of the LMS algorithm with a saturation nonlinearity following the adaptive filter output," IEEE Trans. Signal Process., vol. 49, no. 7, pp. 1370-1387, 2001.

[23] M. H. Costa, J. C. M. Bermudez, and N. J. Bershad, "Statistical analysis of the FXLMS algorithm with a nonlinearity in the secondary-path," Proc. 1999 IEEE International Symposium on Circuits and Systems (ISCAS), pp. III-166-169, 1999.

[24] M. H. Costa, J. C. M. Bermudez, and N. J. Bershad, "Statistical analysis of the Filtered-X LMS algorithm in systems with nonlinear secondary path," IEEE Trans. Signal Process., vol. 50, no. 6, pp. 1327-1342, 2002.

[25] S. D. Snyder and N. Tanaka, "Active control of vibration using a neural network," IEEE Trans. Neural Netw., vol. 6, no. 4, pp. 819-828, 1995.

[26] M. H. Costa, "Theoretical transient analysis of a hearing aid feedback canceller with a saturation type nonlinearity in the direct path," Comput. Biol. Med., vol. 91, pp. 243-254, 2017.
[27] M. H. Costa, L. R. Ximenes, and J. C. M. Bermudez, "Statistical analysis of the LMS adaptive algorithm subjected to a symmetric dead-zone nonlinearity at the adaptive filter output," Signal Process., vol. 88, pp. $1485-1495,2008$

[28] O. J. Tobias and R. Seara, "On the LMS algorithm with constant and variable leakage factor in a nonlinear environment," IEEE Trans. Signal Process., vol. 54, no. 9, pp. 3448-3458, 2006.

[29] N. J. Bershad, "On error-saturation nonlinearities in NLMS adaptation," IEEE Trans. Signal Process., vol. 57, no. 10, pp. 4105-4111, 2009.

[30] H. Hamidi, F. Taringoo, and A. Nasiri, "Analysis of FX-LMS algorithm using a cost function to avoid nonlinearity," Proc. 5th Asian Control Conference, pp. 1168-1172, 2004.

[31] A. Stenger and W. Kellermann, "Nonlinear acoustic echo cancellation with fast converging memoryless preprocessor," Proc. IEEE Int. Conf. Acoustics, Speech, and Signal Processing (ICASSP), pp. 805-808, 2000.

[32] B. Widrow and M. E. Hoff, Jr., "Adaptive switching circuits," IRE WESCON Conv. Rec., Pt. 4, pp. 96-104, 1960.

[33] H. Nishimori, Statistical Physics of Spin Glasses and Information Processing: An Introduction, Oxford University Press, New York, 2001.

[34] S. Miyoshi and Y. Kajikawa, "Statistical-mechanics approach to the Filtered-X LMS algorithm," Electron. Lett., vol. 47, no. 17, pp. 997999, Aug. 2011.

[35] S. Miyoshi and Y. Kajikawa, "Statistical-mechanics approach to theoretical analysis of the FXLMS algorithm, IEICE Trans. Fundam. Electron. Commun. Comput. Sci., vol. E101-A, no. 12, pp. 2419-2433, Dec. 2018.

[36] K. Motonaka, T. Koseki, Y. Kajikawa, and S. Miyoshi, "Statisticalmechanical analysis of adaptive Volterra filter with the LMS algorithm," IEICE Trans. Fundamentals Electron. Commun. Comput. Sci., vol. E104-A, no. 12, pp. 1665-1674, Dec. 2021.

[37] V. J. Mathews, "Adaptive polynomial filters," IEEE Signal Process. Mag., vol. 8, no. 3, pp. 10-26, 1991.

[38] A. Engel and C. V. Broeck, Statistical Mechanics of Learning, Cambridge University Press, Cambridge, 2001.

[39] S. Miyoshi and Y. Kajikawa, "Statistical-mechanical analysis of the FXLMS algorithm with actual primary path," Proc. IEEE Int. Conf. Acoustics, Speech, and Signal Processing (ICASSP), pp. 3502-3506, 2015.

[40] O. J. Tobias, J. C. M. Bermudez, and N. J. Bershad, "Mean weight behavior of the Filtered-X LMS algorithm," IEEE Trans. Signal Process., vol. 48, no. 4, pp. 1061-1075, Apr. 2000.

[41] O. J. Tobias, J. C. M. Bermudez, R. Seara, and N. Bershad, "An improved model for the second moment behavior of the Filtered-X LMS algorithm," Proc. IEEE Adaptive Syst. Signal Process., Commun., Contr. Symp., Lake Louise, AB, Canada, pp. 337-341, Oct. 2000.

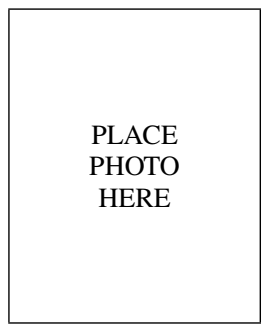

Seiji Miyoshi received his B.Eng. and M.Eng. degrees in electrical engineering from Kyoto University, Japan, in 1986 and 1988, respectively, and his $\mathrm{Ph} . \mathrm{D}$ degree in system science and engineering from Kanazawa University, Japan, in 1998. He worked with the Space Development Division of NEC Corporation from 1988 to 1994 . He joined the Department of Electronic Engineering of Kobe City College of Technology in 1994. He joined the Department of Electrical and Electronic Engineering, Faculty of Engineering Science of Kansai University in 2008, where he is now a professor. His research interests include statisticalmechanical informatics, image processing, signal processing, and learning theory. He is a senior member of the Institute of Electronics, Information and Communication Engineers (IEICE) and the Institute of Electrical and Electronics Engineers (IEEE), and a member of the Physical Society of Japan (JPS) and the Japan Neural Network Society (JNNS). He served as an associate editor of IEICE Transactions on Fundamentals of Electronics, Communications and Computer Sciences from 2013 to 2017 and an awards committee member of the IEEE Kansai section from 2011 to 2014 . He received the 2011 Papers of Editors' Choice from the Journal of the Physical Society of Japan, the Excellent Paper Award in ICONIP2016, and the 2019 Best Paper Award from IEICE. 